\title{
Variation in reproductive strategies of three populations of Phrynocephalus helioscopus in China
}

\author{
Tao Liang ${ }^{1}$, Lu Zhou ${ }^{1,2}$, Wenfeng He ${ }^{1}$, Lirong Xiao ${ }^{1}$, Lei Shi ${ }^{\text {Corresp. } 1}$ \\ ${ }^{1}$ College of Animal Science, Xinjiang Agricultural University, Urumqi, Xinjiang, China \\ 2 Institute of Hydrobiology, Chinese Academy of Science, Wuhan, Hubei, China \\ Corresponding Author: Lei Shi \\ Email address: leis@xjau.edu.cn
}

Background. Egg size and clutch size are key life history traits. During the breeding period, it is possible for females to increase their reproductive output either by increasing the number of eggs if the optimal egg size (OES) is maintained, or by increasing the allocation of energy to each egg. However, the strategies adopted are often influenced by animals' morphology and environment.

Methods. Here, we examined variation in female morphological and reproductive traits, tested for tradeoffs between egg size and clutch size, and evaluated the relationship between egg size and female morphology in three populations of Phrynocephalus helioscopus.

Results. Female body size, egg size, and clutch size were larger in the Yi Ning (YN) and Fu Yun (FY) populations than in the Bei Tun (BT) population (the FY and YN populations laid more, and rounder eggs). Egg size was independent of female body size in two populations (BT and FY), even though both populations had an egg-size/clutch size trade-off. In the YN population, egg size and clutch size were independent, but egg size was correlated with female body size, consistent with the hypothesis of morphological constraint. Conclusions. Our study found geographical variation in body size and reproductive strategies of the lizard Phrynocephalus helioscopus. Egg size was correlated with morphology in the larger-bodied females of the YN population, but not in the smaller-bodied females of the BT population, illustrating that constraints on female body size and egg size are not consistent between populations. 


\section{Variation in Reproductive Strategies of Three Populations of}

\section{Phrynocephalus helioscopus in China}

3 Tao Liang, ${ }^{1}$ Lu Zhou, ${ }^{1,2}$ Wenfeng He,,${ }^{1}$ Lirong Xiao, ${ }^{1}$ Lei Shi ${ }^{1}$

$4 \quad{ }^{1}$ College of Animal Science, Xinjiang Agricultural University, Urumqi, Xinjiang, China

$5 \quad 2$ Institute of Hydrobiology, Chinese Academy of Sciences, Wuhan, Hubei, China

6 Corresponding Author: Lei Shi

$7 \quad$ Email address: leis@xjau.edu.cn

\section{Abstract}

9 Background. Egg size and clutch size are key life history traits. During the breeding period, it is possible for females to increase their reproductive output either by increasing the number of eggs

11 if the optimal egg size (OES) is maintained, or by increasing the allocation of energy to each egg. However, the strategies adopted are often influenced by animals' morphology and environment.

Methods. Here, we examined variation in female morphological and reproductive traits, tested for trade-offs between egg size and clutch size, and evaluated the relationship between egg size and female morphology in three populations of Phrynocephalus helioscopus.

Results. Female body size, egg size, and clutch size were larger in the Yi Ning (YN) and Fu Yun (FY) populations than in the Bei Tun (BT) population (the FY and YN populations laid more, and rounder eggs). Egg size was independent of female body size in two populations (BT and FY), even though both populations had an egg-size/clutch size trade-off. In the YN population, egg size and clutch size were independent, but egg size was correlated with female body size, consistent with the hypothesis of morphological constraint. 
22 Conclusions. Our study found geographical variation in body size and reproductive strategies of

the lizard Phrynocephalus helioscopus. Egg size was correlated with morphology in the largerbodied females of the YN population, but not in the smaller-bodied females of the BT population, illustrating that constraints on female body size and egg size are not consistent between populations.

\section{Background}

Reproductive traits are often variable in animals as a result of differences in the quality of resources and food availability in different habitats (Roff, 2002; Cruz-Elizalde \& RamırezBautista, 2016). Egg size and clutch size are key life history traits, and have received more attention than other reproductive traits (Amat 2008; Qu et al., 2011; Lovich et al., 2012). When there is less food available, females may face the problem of limited available reproductive resources to invest in eggs. This results in a trade-off between 1) the energy allocated to each egg (egg size), and 2) the total number of eggs (clutch size). An increase in resources allocated to each egg will result in a decrease in clutch size (Roff, 1992; Kaplan \& Phillips, 2006). This negative relationship between egg size and clutch size provides evidence for reproductive tradeoffs (Rowe, 1992). Variation in female reproductive output is widespread, both interspecifically and intraspecifically. Especially for geographically widespread species, local genetic variation, short-term phenotypic plasticity, and the complex interactions between these two, contribute to variation in reproductive output (Brown \& Shine, 2007).

Optimal egg size (OES) theory predicts that natural selection optimizes egg size within populations, such that when resources are not limiting for reproduction, clutch size or number of 
clutches may increase, while egg size remains constant (Smith \& Fretwell, 1974; Brockelman, 1975). Natural selection predicts that females should optimize resources allocated to each egg, and clutch size should only increase after ensuring the production of high quality offspring (Lovich et al., 2012). In some reptiles, clutch size is positively correlated with female body size, while egg size remains constant, consistent with OES theory (Congdon \& Gibbons, 1987). However, the relationship between egg size and clutch size is determined by many factors, and the trade-offs between egg size and clutch size are not always evident in natural populations (Berven, 1982; Liao \& Lu, 2011; Wang et al., 2011).

Egg size is often correlated with female body size in reptiles (morphological constraint hypothesis), and both egg size and clutch size increase with an increase in female body size, contrary to OES theory (Dunham \& Miles, 1985; Clark, Ewert \& Nelson, 2001; Ryan \& Lindeman, 2007; Mohamed et al., 2012). When resources are limited, reproductive output is directly correlated with the trade-offs between egg size and clutch size, and ultimately, with offspring survival (Congdon \& Tinkle, 1982; Brown \& Shine, 2009). The size of each egg normally determines the success of incubation and offspring survival (Angilletta et al., 2004; Räsänen, Laurila \& Merilä, 2005). Females may increase energy allocation to eggs as a way to improve offspring quality.

Phrynocephalus helioscopus are small (mean snout-vent length (SVL): $47.5 \mathrm{~mm}$ ) lizards that are widely distributed in Eurasia. Previous research on this species has focused on egg incubation (Wang et al., 2013), and female reproductive output (Liang et al., 2015). However, variation in female reproductive traits, and egg size-clutch size trade-offs, and the effects of 
64 female size on egg size have not been studied in geographically different populations in order to understand variation in investment in offspring production. In this study, we compared female morphological traits and the relationships between egg length (EL), egg width (EW), egg mass (EM), egg shape (ES), and clutch size (CS) in three populations of $P$. helioscopus. Specifically, we

1. Tested whether reproductive female size differed between the three populations, and 2. Examined how that variation was associated with reproductive traits, especially fecundity, egg and clutch size, egg shape, and the trade-offs between egg size and clutch size.

\section{Materials and Methods}

\subsection{Ethical approval}

Specimens were collected following Guidelines for Use of Live Amphibians and Reptiles in Field Research (the Herpetological Animal Care and Use Committee (HACC) of the American Society of Ichthyologists and Herpetologists, 2004). This study was conducted in compliance with current laws on animal welfare and research in China. After the research was completed, lizards were released where they were captured.

\subsection{Study site}

The populations studied here were located in three ecologically distinct locations in the Xinjiang Uyghur Autonomous Region, China, and included locations near Bei Tun city (BT:

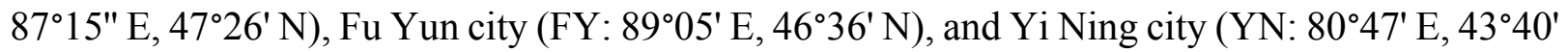
$\mathrm{N})$. The BT and the YN populations were located approximately $660 \mathrm{~km}$ apart, and the habitats were different at each location. The BT population was located in a typical gravel desert with 
little vegetation, while the YN population was located in a loam desert with abundant vegetation.

The climate experienced by the YN population was hotter and wetter than that experienced by the BT population. The FY and BT populations were located approximately $160 \mathrm{~km}$ apart, and the FY and YN populations were located approximately $700 \mathrm{~km}$ apart. The vegetation and rainfall at the FY and YN locations were similar, while the temperature regimes were similar at the FY and BT locations (Figs. 1, 2).

\subsection{Animal and egg collection}

In May 2014 and May 2017, we collected P. helioscopus by hand from locations near BT (in 2014, Liang et al., 2015), FY (in 2017), and YN (in 2017). We collected lizards from 12:00 to $18: 00$, when they were most active (personal observation), and we captured the lizards at random. Lizards were transported to Xinjiang Agricultural University, where female lizards were palpated to determine their reproductive state (Li et al., 2006). Fifty-three gravid females (BT: 13, FY: 24, YN: 16) were housed individually in plastic cages containing vermiculite in a room with ambient temperatures that varied from $20-28{ }^{\circ} \mathrm{C}$ with a 12 -hour light/12-hour dark cycle.

A $250 \mathrm{~W}$ light bulb was suspended at one end of each cage, $20 \mathrm{~cm}$ above the cage floor, and lizards could freely move to warmer and cooler places within the cage. Mealworms (larvae of Tenebrio molitor) and water enriched with vitamins and minerals were provided ad libitum. Females dig before they lay eggs, and we observed females every two hours, which allowed us to collect eggs quickly, and prevented eggs from absorbing water in the moist vermiculite. All eggs used in this study were collected no more than 20 minutes after they were laid.

\subsection{Morphology and reproductive traits}



indicate increasingly elongate eggs (Ji \& Wang, 2005; Kratochvíl \& Frynta, 2006).

\subsection{Statistical analyses}

We used analysis of variance (ANOVA) to examine differences in SVL, EM, and ES, and

we used analysis of covariance (ANCOVA) to examine differences in TBW, EL, EW, RCM, and

CS between the three populations with post hoc Tukey's tests (multiple comparisons). We used

Levene's tests to test for heterogeneity of variances. The residuals were tested by Kolmogorov-

Smirnov tests for detecting normality. We log-transformed the variables to minimize the heterogeneity, where necessary (King, 2000). To test egg size-clutch size trade-offs, and analyze potential morphological constraints on optimal egg size, the relationships between EM and EL and CS, EM and EL, EL and CS and SVL, and EW and TBW were examined using reduced major axis (RMA) regressions rather than ordinary least squares (OLS) regressions, because

RMA accounts for an error in the independent variable (Dunham \& Miles, 1985). Historical climatic data (1990 - 2013) of the three study areas were taken from the Chinese National

125 (calculated using the effect function in the "effects" package, Fox \& Hong 2009) \pm standard error

126 (SE), except for SVL, EM, and ES, which are presented as the mean \pm SE. Differences were 
127

128

129

130

131

132

133

134

135

136

137

138

139

140

141

142

143

144

145

146

147

considered significant when $P<0.05$.

All analyses were conducted using R v. 3.4.1 (R Core Team 2017), using the packages

"lmodel2” (Legendre, 2011), “ggplot2” (Wickham 2015), and "gplots” (Warnes et al., 2011).

\section{Results}

\subsection{Female morphological variation}

SVL varied between populations, and was longest in the YN and FN populations, in which females had similar SVLs (YN: $51.23 \mathrm{~mm}$; FY: $50.43 \mathrm{~mm}$ ), and shortest in the BT population $\left(F_{2,52}=20.75, r^{2}=0.45, P<0.0001\right.$, Fig. 3A $)$. TBW varied between populations, and was smallest in the YN and FN populations, which had similar TBWs (YN: $7.20 \mathrm{~mm}$; BT: $6.93 \mathrm{~mm}$ ), and largest in the FY population when controlling for SVL using $\operatorname{ANCOVA}\left(F_{2,52}=6.82, P=0.002\right.$, Fig. 3B).

\subsection{Female reproductive traits}

Females in the FY population laid heavier eggs than those in the BT and YN populations (Table 1). Eggs were similar in length in all three populations. Eggs were wider in the FY population, and narrower in the YN population. BT females laid smaller clutches than FY and YN females when controlling for SVL (Table1).

\subsection{Egg size-clutch size trade-offs}

We found a positive relationship in all populations between EL and EM (Fig. 4C). In BT and FY populations, egg size decreased with clutch size, while in $\mathrm{YN}$ females, egg size was independent of clutch size (Fig. 4 A, B).

\subsection{The relationship between egg size, clutch size, and female morphology}


In the BT and YN populations, female body size was independent of EL, EW, and CS (Fig.

\section{Discussion}

Females in our three populations varied in body size (SVL, TBW), and reproductive traits

(EM, CS, RCM, and egg size), in ways that supported, with some exceptions, an egg size-clutch

size trade-off in these three populations of $P$. helioscopus. Female body size, egg size, and clutch

size were smaller in the BT population than in the FY and YN populations, and the FY and YN

populations laid more, and rounder eggs. Egg size was not correlated with female body size in the

BT and FY populations, but egg size-clutch size trade-offs occurred in both populations. Trade-

offs between egg size and clutch size were not found in the YN population, but egg size was correlated with female body size in this population.

Morphological traits, such as body size and body shape, always vary among different

populations of animals (e.g. snakes: Zhong et al., 2017; lizards: Horváthová et al., 2013; turtles:

Werner et al., 2016). Environmental factors that exert strong effects on animal life history traits include activity season length and food availability (Yom-Tov et al., 2006; Horváthová et al., 2013). Our study revealed that females in the FY and YN populations were larger than females in the BT population. Longer activity seasons were assumed to be the cause of variation in the body size between the females of the YN and BT populations (Liang \& Shi, 2017). Temperature, which is fundamentally important for lizards (Grant \& Dunham 1990), was highest in YN, especially in 
169 hibernation began in early November, which means that the activity period for lizards in $\mathrm{YN}$ was 170 almost a month longer than in the other two sites (BT and FY). P. helioscopus in this population 171 were larger, probably because of the longer growing season. It is possible that $P$. helioscopus age 172 varied between the three study locations, and this may have influenced results. However, we have 173 no reason to believe that the ages of the $P$. helioscopus captured from the three populations were 174 very different. We could have tested for this possibility using mark and recapture methods, but unfortunately, this was outside the scope of this study.

The BT and the FY populations experienced similar temperatures, which raises the question as to what caused the differences in body size. One possibility is that food limitation might have resulted in reduced growth rates in the BT population. Rainfall is critical for habitat quality (e.g. vegetation cover and prey abundance, Lorenzon, Clobert \& Massot, 2001) and there was 180 significant geographic variation in rainfall between BT and FY (Fig. 2). BT is sparsely vegetated, due to drier conditions in this location, whereas the vegetation is more abundant in the wetter FY and YN sites (Fig. 1, Fig. 2B). Humidity is the most important factor influencing abundance and distribution of insects (Savopoulou-Soultani et al., 2012; Cesne, Wilson \& Soulier-Perkins, 2015), and therefore drier conditions and sparse vegetation could be associated with less available food in the BT population.

Egg size varies between populations because of variation in female body size, which is an important female trait that can affect offspring quality (Steyermark \& Spotila, 2001; Morrison \& Hero, 2003; Olsen \& Vollested, 2003). We found that egg size differed between the three populations, which suggests that larger females in the FY and YN populations were able to allocate 
190

191

192

193

194

195

196

197

198

199

200

201

202

203

more resources to egg production. In addition, egg size is also correlated with the incubation

period, with smaller eggs having a relatively short incubation time. However, further studies are

need to determine whether the earlier hatching of the smaller eggs in the BT population could provide offspring with more time to forage before the hibernation period (Thompson \& Pianka, 2001). EL was similar in the three populations (using EM as the covariate), but EW was not. However, egg shape is also related to clutch size, and larger clutches tend to have more rounded eggs (Ji et al., 2002). Eggs were narrower in the BT population, and both the FY and YN populations laid more, and rounder eggs (Table 1). Larger reptile females tend to lay more eggs (Ryan \& Lindeman, 2007; Amat, 2008). Thus, the smaller CS of the BT population, associated with their smaller body size, may be due to limited resources (including food), as resource availability often varies between populations (Liao, Lu \& Jehle, 2014; Roitberg et al., 2015).

The trade-off between egg size and clutch size is an important concept in life-history theory (Kern et al., 2015). Egg size (EM and EL) and clutch size were negatively correlated in the BT and FY populations, but not in the YN population. In the $\mathrm{YN}$ population, there was no egg sizeclutch size trade-off, suggesting that patterns of variation in egg size and clutch size are not always consistent in different populations (Liao, Lu \& Jehle, 2014; Roitberg et al., 2015).

Generally speaking, offspring phenotypes are influenced by female body size (e.g., SVL, Krist \& Remes, 2004). Body size and other factors affecting egg size will result in the following five possible outcomes (Lovich et al., 2012): 1) egg size is constrained by female size (not optimized), 2) egg size is unconstrained by female morphology (optimized), 3) egg size is unconstrained by female morphology and optimized only in the largest females (Fehrenbach et al., 
211 2016), 4) egg size is not constrained by the pelvic aperture width, and is not optimized, but rather

212 is constrained by some other non-morphological factor (e.g., female age or clutch number, Clark,

213 Ewert \& Nelson, 2001; Harms et al., 2005; Paitz et al., 2007), or 5) egg width is constrained and

214 requires osteokinesis for oviposition (Hofmeyr, Henen \& Loehr, 2005; Fehrenbach et al., 2016).

215 Consistent with the prediction of the morphological constraint hypothesis, egg size increased as the size of the female increased (outcome 1) in the YN population. Although female body size in the BT population was smaller than in the FY population, in both cases, egg size was not correlated with female body size (outcome 2 or 4 above). For some species with small body sizes, egg size is constrained by female morphology (Ryan \& Lindeman, 2007). In small-bodied females, the body size-specific constraints on egg size, coupled with selection towards an optimum egg size, results in a positive correlation between body size and egg size. Egg size (EL and EW) was not dependent on female body size in either the BT or FY population, but there were negative correlations between egg size and clutch size (Fig. 4), suggesting that egg size was constrained by CS (a non-morphological factor) in both populations (Brown \& Shine, 2009, outcome 4). Unexpectedly, we found that egg size was correlated with body size in the larger-bodied females of the YN population. A positive relationship between egg size and female size indicates that there is no optimal egg size in the YN population (Escalona, Adams \& Valenzuela, 2018). However, we found some support for the prediction that EW was constrained by TBW (Fig. 3), since eggs must fit the female tail base width, which they pass through on their smallest axis (i.e. EW). In some turtle species, EW but not EL increases with the size of the female (Rasmussen \& Litzgus, 2010). 
232 population, suggesting that EL is dependent upon on female SVL. EL can be constrained by

233 morphological factors, non-morphological factors (e.g. CS), or their interactions, which may

234 indicate that a weak relationship exists between female morphology and EL in the YN population.

235 The specific mechanisms of the non-morphological factors require further study (Kern et al., 236 2015).

\section{5. Conclusions}

We found geographic variation in body size and reproductive strategies of $P$. helioscopus.

Females in populations with longer growing seasons and abundant vegetation (the FY and YN

populations) were larger. Lizards in the BT population were smaller, perhaps due to limitations on

food availability, or limitations on activity seasons, and also had smaller clutches than the FY and

YN populations. Females in the FY and YN populations produced rounder eggs, perhaps due to

their larger body size. This study found that egg size was correlated with female body size in the

larger-bodied females of the YN population - an anomaly for the morphological constraint

hypothesis. Egg size was not correlated with female body size, and did not follow the optimal egg

size hypothesis in the BT and FY populations. Trade-offs between egg size and clutch size suggest that egg size was constrained by clutch size in both populations.

Potential genetic and age variation associated with females in these populations may have influenced our results. However, here we demonstrate that life histories, as measured by body size and clutch characteristics, can vary in surprising ways, sometimes supporting the possibility of trade-offs, and sometimes not.

\section{Acknowledgments}


Southwest Biological Science Center, and anonymous reviewers for their excellent suggestions for improving this manuscript. We thank Luo D and Wang P for assistance during fieldwork, and we

professional advice regarding spelling and phrasing.

258

259

260

261

262

263

264

265

266

267

268

269

270

271

\section{REFERENCES}

Amat F. 2008. Exploring female reproductive tactics: trade-offs between clutch size, egg mass and newborn size in lacertid lizards. Herpetological Journal 18(3): 147-153.

Angilletta MJ, Niewiarowski PH, Dunham AE, Leaché AD, Porter WP. 2004. Bergmann's clines in ectotherms: illustrating a life-history perspective with Sceloporine lizards. The American Naturalist 164(6): E168-E183 DOI: 10.1086/42522.

Brockelman W. 1975. Competition, fitness of offspring, and optimal clutch size. American Naturalist 109(970): 677-699 DOI: 10.1086/283037.

Brown G, Shine R. 2007. Rain, prey and predators: climatically driven shifts in frog abundance modify reproductive allometry in a tropical snake. Oecologia 154(2): 361-368 DOI: $10.1007 / \mathrm{s} 00442-007-0842-8$.

Brown GP, Shine R. 2009. Beyond size-number trade-offs: clutch size as a maternal effect. Philosophical Transactions of the Royal Society B Biological Sciences 364(1520): 1097-1106 DOI: $10.1098 /$ rstb.2008.0247. 
272 Cesne ML. Wilson S W. Soulier-Perkins A. 2015. Elevational gradient of Hemiptera

273 (Heteroptera, Auchenorrhyncha) on a tropical mountain in Papua New Guinea. PeerJ 3(23)

274 DOI: $10.7717 /$ peerj.978

275

Clark PJ, Ewert MA, Nelson CE. 2001. Physical apertures as constraints on egg size and shape in the common musk turtle, Sternotherus odoratus. Functional Ecology 15(1): 70-77 DOI: $10.2307 / 826569$

Congdon JD, Tinkle DW. 1982. Energy expenditure in free-ranging sagebrush lizards (Sceloporus graci). Canadian Journal of Zoology 60(6): 1412-1416 DOI: 10.1139/z82-190.

Congdon JD, Gibbons JW. 1987. Morphological constrains on egg size: a challenge to optimal egg size theory? Proceedings of the National Academy of Sciences of the United States of America 84(12): 4145-4147.

Cruz-Elizalde R, Ramırez-Bautista A. 2016. Reproductive cycles and reproductive strategies among populations of the Rose-bellied lizard Sceloporus variabilis (Squamata: Phrynosomatidae) from central Mexico. Ecology and Evolution 6(6):1753-1768 DOI: 10.1002/ece3.1998.

Dunham AE, Miles DB. 1985. Patterns of covariation in life history traits of squamate reptiles: the effects of size and phylogeny reconsidered. The American Naturalist 126(2): 231-257 DOI: $10.1086 / 284411$

Escalona T, Adams DC, Valenzuela N. 2018. A lengthy solution to the optimal propagule size problem in the large-bodied South American freshwater turtle, Podocnemis unifilis. Evolutionary Ecology 32(1): 29-41 DOI: 10.1007/s10682-017-9922-3 
293 Fehrenbach AK, Louque I, McFadden SL, Huntzinger C, Lyons E, Shively SH, Selman W,

294 Lindeman PV. 2016. Habitat-related variation in body size and reproductive output and an examination of reproductive allometry in the Sabine map turtle (Graptemys sabinensis) across three river drainages. Copeia 104(2):458-468 DOI: 10.1643/CE-15-273.

Fox J, Hong J. 2009. Effect displays in R for multinomial and proportional-odds logit models: Extensions to the effects package. Journal of Statistical Software 32:1: 1-24.

Grant BW, Dunham AE. 1990. Elevational covariation in environmental constraints and life histories of the desert lizard Sceloporus merriami. Ecology 71(5):1765-1776 DOI: $10.2307 / 1937584$

Harms HK, Paitz RT, Bowden RM, Janzen FJ. 2005. Age and season impact resource allocation to eggs and nesting behavior in painted turtles. Physiological and Biochemical Zoology 78(6): 996-1004 DOI: 10.1086/432920.

Hofmeyr MD, Henen BT, Loehr VJT. 2005. Overcoming environmental and morphological constraints: Egg size and pelvic kinesis in the smallest tortoises, Homopus signatus. Canadian Journal of Zoology 83(10): 1343-1352 DOI: 10.1139/Z05-132.

Horváthová T, Cooney CR, Fitze PS, Oksanen T, Jelić D, Ghira I, Uller, Jandzik D. 2013. Length of activity season drives geographic variation in body size of a widely distributed lizard. Ecology \& Evolution 3(8): 2424-2442 DOI: 10.1002/ece3.613.

Ji X, Huang HY, Hu XZ, Du WG. 2002. Geographic variation in female reproductive characteristics and egg incubation in the Chinese skink, Eumeces chinensis. Chinese Journal 
314 Ji X, Wang ZW. 2005. Geographic variation in reproductive traits and trade-offs between size and

315 number of eggs of the Chinese cobra (Naja atra). Biological Journal of the Linnean Soeiety 85(1): 27-40 DOI: $10.1002 /$ ece3.613.

317

318

319

320

321

322

323

324

325

326

327

328

329

330

331

332

333

334

Kaplan RH, Phillips PC. 2006. Ecological and developmental context of natural selection: maternal effects and thermally induced plasticity in the frog Bombina orientalis. Evolution 60(1): 142-156 DOI: 10.1111/j.0014-3820.2006.tb01089.x.

Kern MM, Guzy JC, Lovich JE, Gibbons JW, Dorcas ME. 2015. Relationships of maternal body size and morphology with egg and clutch size in the Diamondback terrapin, Dalaclemys terrapin (Testudines: Emydidae). Biological Journal of the Linnean Society 117(2): 295-304 DOI: $10.1111 /$ bij.12655.

King RB. 2000. Analyzing the relationship between clutch size and female body size in reptiles. Journal of Herpetology. 34(1): 148-150 DOI: 10.1111/j.0014-3820.2006.tb01089.x.

Kratochvíl L, Frynta D. 2006. Egg shape and size allometry in geckos (Squamata: Gekkota), lizards with contrasting eggshell structure: why lay spherical eggs? Journal of Zoological Systematics and Evolutionary Research 44(3): 217-222 DOI: 10.1111/j.1439-0469.2005.00339.x.

Krist M, Remeš V. 2004. Maternal effects and offspring performance: in search of the best method. Oikos 106(2): 422-426 DOI: 10.1111/j.0030-1299.2004.13373.x.

Legendre P. 2011. lmodel2: Model II Regression. R package version 1.7-1/ r1794. http://RForge.R-project.org/projects/vegan/

Li H, Ji X, Qu YF, Gao JF, Zhang L. 2006. Sexual dimorphism and female reproduction in the multi-ocellated racerunner Eremias multiocellata (Lacertidae). Acta Zoologica Sinica 52(2): 
335

336

337

338

339

340

341

342

343

344

345

346

347

348

349

350

351

352

353

354

250-255.

Liang T, LÜ SP, Wu KL, Shi L. 2015. Sexual dimorphism and female reproduction of Phrynocephalus helioscopus (Agamidae). Chinese Journal of Ecology 34(6): 1602-1606 DOI: 10.13292/j.1000-4890.2015.0144 (Abstract by English).

Liang T, Shi L. 2017. Sexual dimorphism and morphological variation of three populations of Phrynocephalus helioscopus: Test of Bergmann's rule, Allen's rules and Rensch's rule. Sichuan Journal of Zoology 36(3): 249-257 DOI: 10.11984/j.issn.1000-7083.20160314 (Abstract by English)

Liao WB, Lu X. 2011. A comparison of reproductive output of the Omei Treefrog (Rhacophorus omeimontis) between high and low elevations. Animal Biology 61(3): 263-276 DOI: $10.1163 / 157075511 X 584218$.

Liao WB, Lu X, Jehle R. 2014. Altitudinal variation in maternal investment and trade-offs between egg size and clutch size in the Andrew's toad. Journal of Zoology 293 (2): 84-91 DOI: $10.1111 /$ jzo.12122.

Lorenzon P, Clobert J, Massot M. 2001. The contribution of phenotypic plasticity to adaptation in Lacerta vivipara. Evolution 55: 392-404 DOI: $10.1554 / 0014-$ 3820(2001)055[0392:TCOPPT]2.0.CO;2

Lovich JE, Madrak SV, Drost CA, Monatesti JA, Casper D, Znari M. 2012. Optimal egg size in a suboptimal environment: reproductive ecology of female Sonora mud turtles (Kinosternon sonoriense) in central Arizona, USA. Amphibia-Reptilia 33(2):161-170 DOI: 
356

357

358

359

Mohamed N, Znari M, Lovich JE, Feddadi Y, Baamrane MAA. 2012. Clutch and egg allometry of the turtle Mauremys leprosa (Chelonia: Geoemydidae) from a polluted periurban river in west-central Morocco. Herpetological Journal 22(1):43-49.

Morrison C, Hero JM. 2003. Geographic variation in lifehistory characteristics of amphibians: a review. Journal of Animal Ecology 72(2): 270-279 DOI: 10.1046/j.1365-2656.2003.00696.x.

Olsen EM, Vøllested LA. 2003. Microgeographical variation in brown trout reproductive traits: possible effects of biotic interactions. Oikos 100(3): 483-492 DOI: 10.1034/j.16000706.2003.11900.x.

Paitz RT, Harms HK, Bowden RM, Janzen FJ. 2007. Experience pays: offspring survival increases with female age. Biology Letters 3(3): 44-46 DOI: 10.1098/rsbl.2006.0573.

Qu YF, Li H, Gao JF, Ji X. 2011. Geographical variation in reproductive traits and trade-offs between size and number of eggs in the king ratsnake, Elaphe carinata. Biological Journal of the Linnean Society 104(3): 701-709 DOI: 10.1111/j.1095-8312.2011.01749.x.

Räsänen K, Laurila A, Merilä J. 2005. Maternal investment in egg size: environment and population-specific effects on offspring performance. Oecologia 142(4): 546-553 DOI: $10.1007 / \mathrm{s} 00442-004-1762-5$.

Rasmussen M, Litzgus J. 2010. Patterns of maternal investment in spotted turtles (Clemmys guttata): implications of trade-offs, scales of analyses, and incubation substrates. Écoscience 17(1):47-58 DOI: $10.2980 / 17-1-3298$.

Roff DA. 1992. The evolution of life histories. New York: Chapman \& Hall. Roff DA. 2002. Life-history evolution. Sunderland: Sinauer Associates. 
377 Roitberg ES, Eplanova GV, Kotenko TI, Amat F, Carretero MA, Kuranova VN, Bulakhova NA,

378 Zinenko OI, Yakovlev VA. 2015. Geographic variation of life-history traits in the sand lizard, 379 Lacerta agilis: testing Darwin's fecundity-advantage hypothesis. Journal of Evolution Biology 28(3): 613-629 DOI: 10.1111/jeb.12594.

381

382

383

384

385

386

387

388

389

390

391

392

393

394

395

396

397

Rowe J. 1992. Comparative life histories of the painted turtle (Chrysemys picta) from Western Nebraska. Dissertation, University of Nebraska-Lincoln, University of Nebraska-Lincoln.

Ryan KM, Lindeman PV. 2007. Reproductive allometry in the Common Map Turtle Graptemys geographica. American Midland Naturalist 158(1): 49-59 DOI: 10.1674/00030031(2007)158[49:RAITCM]2.0.CO;2.

Savopoulou-Soultani M, Papadopoulos NT, Milonas P, Moyal P. 2012. Abiotic factors and insect abundance. Psyche 2012:1-2 DOI 10.1155/2012/167420.

Smith C, Fretwell S. 1974. Optimal balance between size and number of offspring. American Naturalist 108(962): 499-506 DOI: 10.1086/282929.

Steyermark AC, Spotila JR. 2001. Body temperature and maternal identity affect snapping turtle (Chelydra serpentina) righting response. Copeia 2001(4): 1050-1057 DOI: http://dx.doi.org/10.1643/0045-8511

Thompson GG, Pianka ER. 2001. Allometry of clutch and neonate sizes in Monitor lizards (Varanidae: Varanus). Copeia 2001(2): 443-458 DOI: 10.1643/0045-8511(2001)001.

Wang YJ, Ji WH, Zhao W, Nannan Y, Liu NF. 2011. Geographic variation in clutch and egg size for the lizard Phrynocephalus przewalskii (Squamata: Agamidae). Asian Herpetological Research 2(2): 97-102 DOI: 10.3724/SP.J.1245.2011.00097. 
398

399

400

401

402

403

404

405

406

407

408

409

410

411

412

413

414

415

Wang Z, Ma L, Shao M, Ji X. 2013. Differences in incubation length and hatchling morphology among five species of oviparous Phrynocephalus lizards (Agamidae) from China. Aisan Herpetological Research 4(4): 225-232 DOI: 10.3724/SP.J.1245.2013.00225.

Warnes GR, Bolker B, Bonebakker L, Gentleman R, Liaw WHA, Lumley T, Maechler M, Magnusson A, Moeller S, Schwartz M, Venables B. 2011. gplots: Various R programming tools for plotting data. The Comprehensive Archive Network. https://cran.rproject.org/web/packages/gplots/gplots.pdf.

Werner YL, Korolker N, Sion G, Göçmen B. 2016. Bergmann's and Rensch's rules and the spurthighed tortoise (Testudo graeca). Biological Journal of the Linnean Society 117(4):796-811 DOI: $10.1111 /$ bij.12717.

Wickham H. 2015. ggplot2. Wiley Interdisciplinary Reviews Computational Statistics, 3(2):180185.

Yom-Tov Y, Heggberget TM, Øystein W, Yom-Tov S. 2006. Body size changes in the Norwegian otter: the possible effects of food availability and global warming. Oecologia 150(1):155-160 DOI: $10.1007 / \mathrm{s} 00442-006-0499-8$.

Zhong GH, Liu Q, Li C, Peng P, Guo P. 2017. Sexual dimorphism and geographic variation in the asian lance-headed pitviper Protobothrops mucrosquamatus in the mainland china. Asian Herpetological Research 8(2): 118-122 DOI: 10.16373/j.cnki.ahr.160011. 
Figure 1

Figure 1. Map showing the three locations in the Xinjiang Uyghur Autonomous Region of western China where Phrynocephalus helioscopus were captured for this study.

The nearest cities (BT: Bei Tun, FY: Fu Yun, YN: Yi Ning) are identified by the red dots, and the collection locations are indicated by the black dots with arrows. Photos indicate habitat types in each sampling location (Photo credit: Tao Liang).

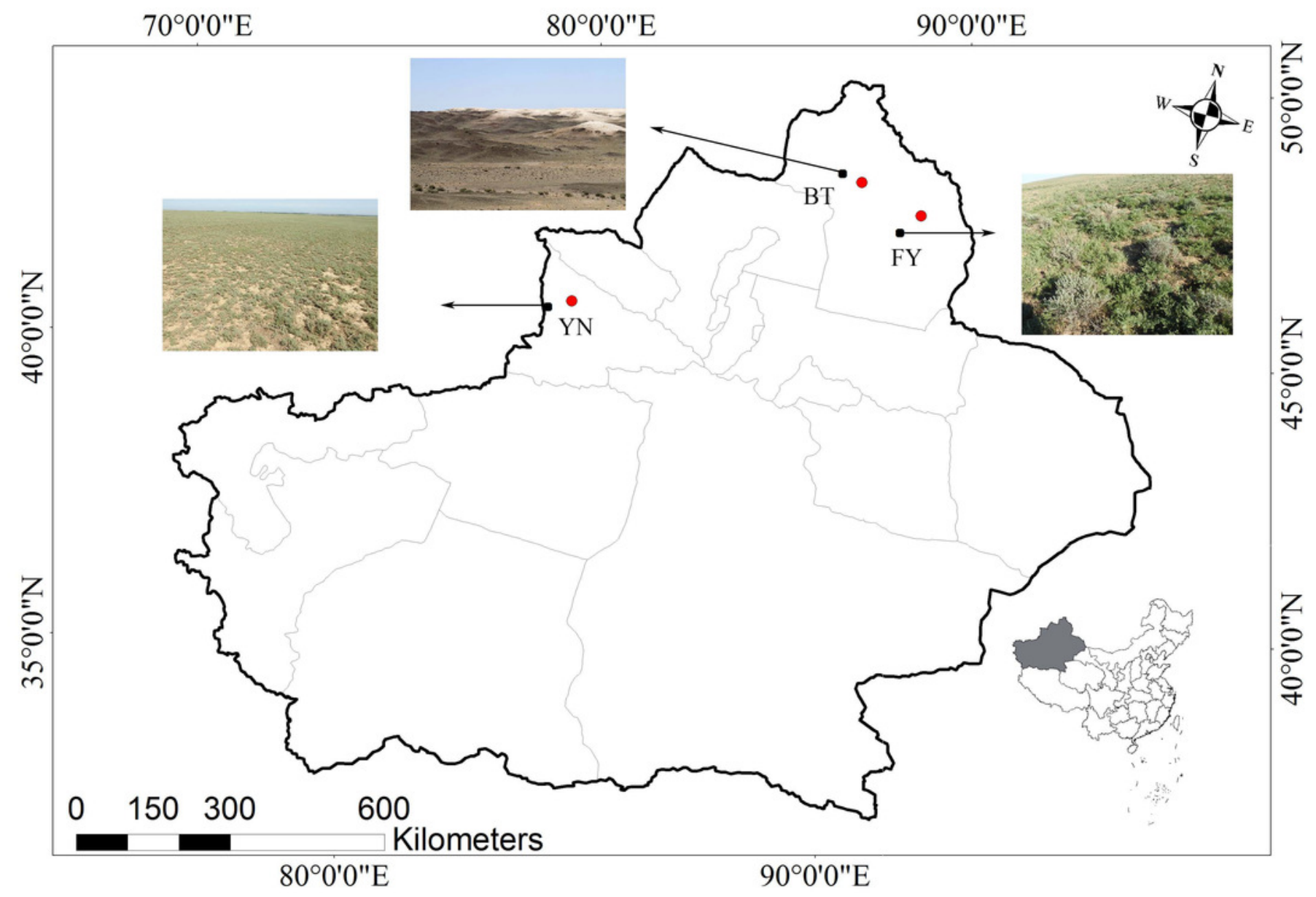


Figure 2

Figure 2. Means for monthly mean air temperature $(A)$ and monthly mean rainfall (B) from 1990 to 2013 at the three sampling locations (BT: Bei Tun, FY: Fu Yun, YN: Yi Ning), where Phrynocephalus helioscopus were collected.

Note that months are numbered from 1 (January) to 12 (December).
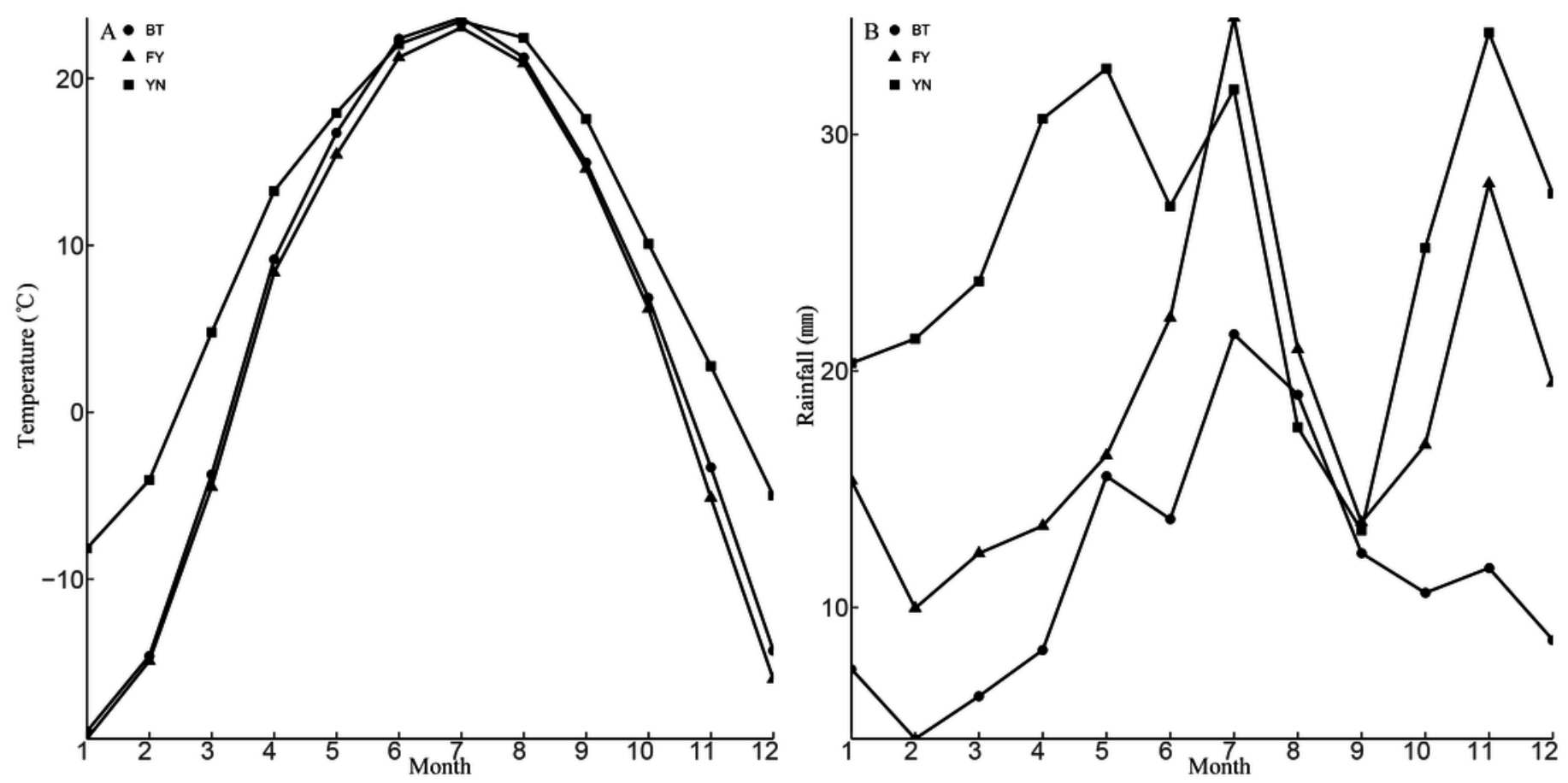
Figure 3

Figure 3. Comparisons between A) snout-vent length and B) tail width at base, of gravid females in three populations (BT: Bei Tun, FY: Fu Yun, YN: Yi Ning) of Phrynocephalus helioscopus.

Points indicate means with $95 \%$ confidence intervals. Different letters indicate significant differences at the $P<0.05$ level.
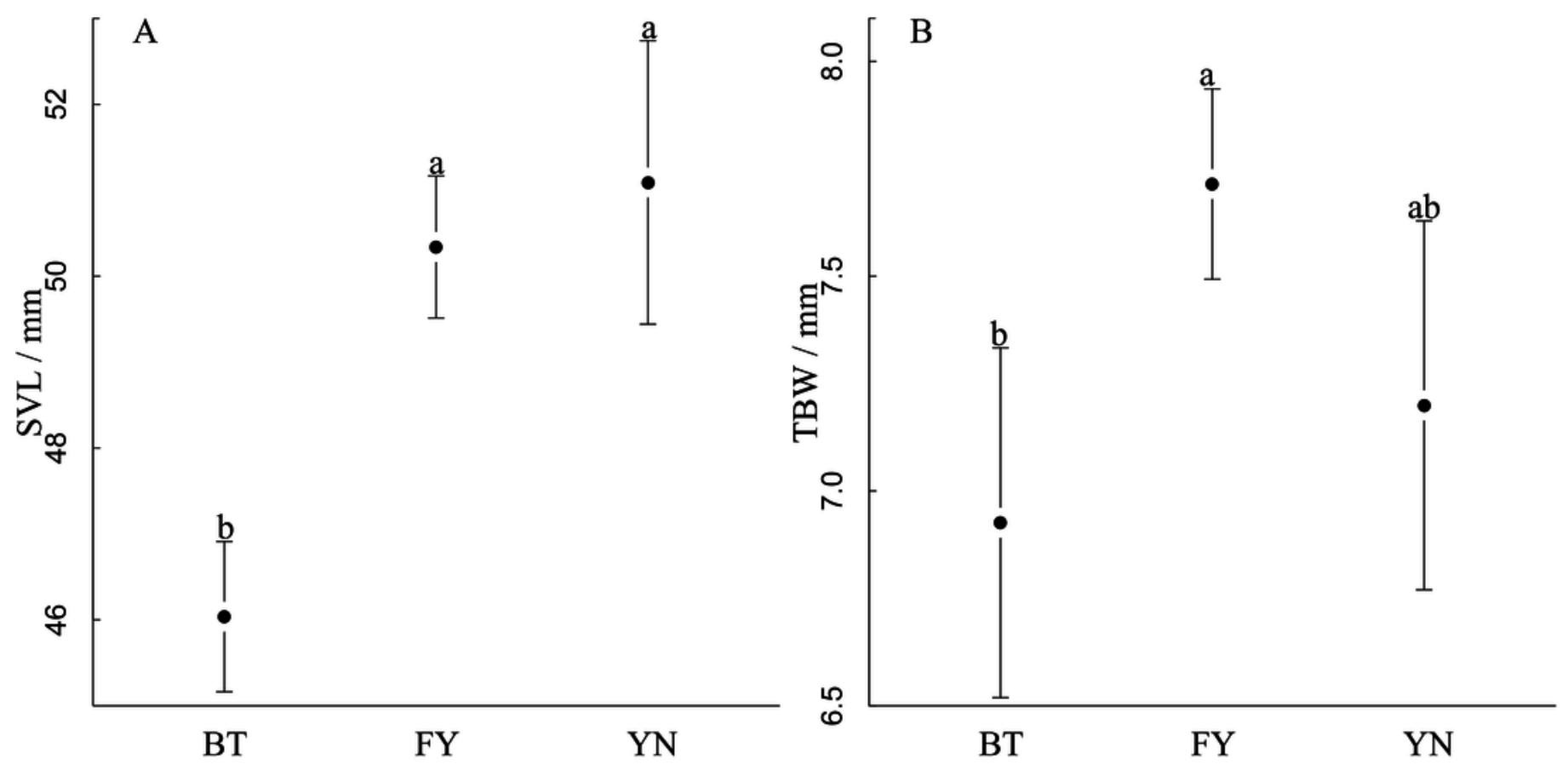
Figure 4

Figure 4. Regressions of egg length (EL) and egg mass (EM) and clutch size (CS) tradeoffs in Phrynocephalus helioscopus.

Fitted reduced major axis regression model and statistical significance $(P<0.05)$ are indicated in each case. BT: Bei Tun - Shaded circle , FY: Fu Yun - Shaded triangle , YN: Yi Ning - Shaded square. Points were jittered by geom jitter function.
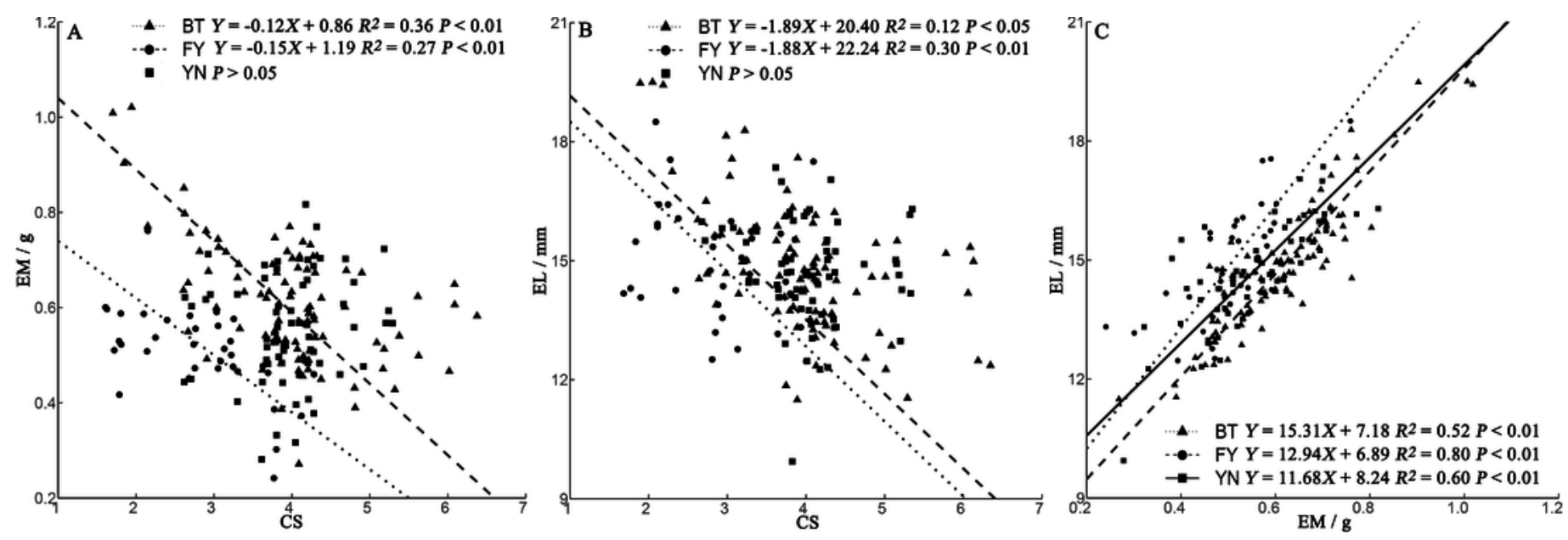
Figure 5

Regressions of egg length (EL) (A), egg width (EW) (B), and clutch size (CS) (C) and female morphological traits (SVL: snout-vent length, TBW: tail base width) from three populations of Phrynocephalus helioscopus.

Fitted reduced major axis regression model and statistical significance $(P<0.05)$ are indicated in each case. BT: Bei Tun - Shaded circle , FY: Fu Yun - Shaded triangle , YN: Yi Ning

- Shaded square. Points were jittered by geom jitter function.
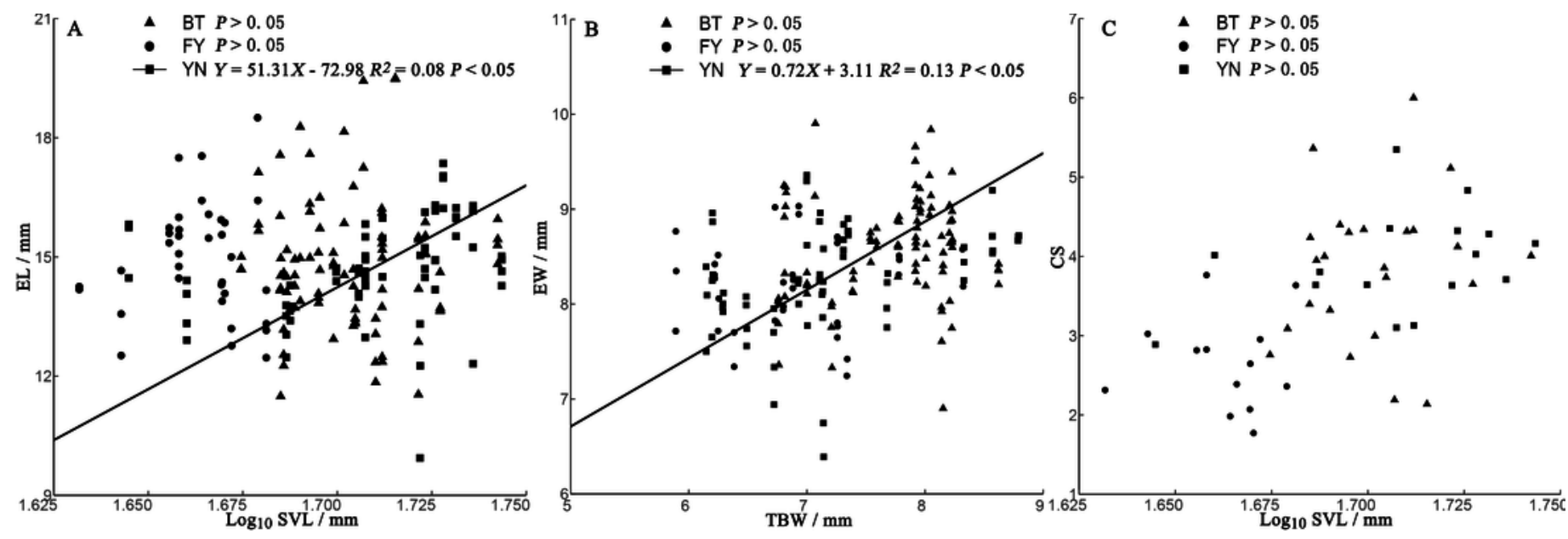


\section{Table $\mathbf{1}$ (on next page)}

Table 1. Descriptive statistics of female reproductive traits in the three populations (BT: Bei Tun, FY: Fu Yun, YN: Yi Ning) of Phrynocephalus helioscopus. EM: egg mass, EL: egg length, EW: egg width, ES: egg shape, CS: clutch size.

Notes: Different letters indicate significant at the $P<0.05$ level;

† ANOVA; \# One-way analyses of covariance (ANCOVAs) (for CS with snout-vent length (SVL) as the covariate, and for EL and EW with egg mass (EM) as the covariate);

$* \mathrm{BT} n=13, \mathrm{FY} n=24, \mathrm{YN} n=16$. 


\begin{tabular}{|c|c|c|c|c|}
\hline & $\mathrm{BT}(\mathrm{n}=35)$ & $\mathrm{FY}(\mathrm{n}=90)$ & $\mathrm{YN}(\mathrm{n}=63)$ & $F$-level and $P$-value \\
\hline $\mathrm{EM}(\mathrm{g})^{\dagger}$ & $0.51 \pm 0.02^{b}$ & $0.61 \pm 0.02^{\mathrm{a}}$ & $0.55 \pm 0.01^{b}$ & $F_{2,187}=11.67, r^{2}=0.11, P<0.0001$ \\
\hline range & $0.32 \sim 0.76$ & $0.27 \sim 1.02$ & $0.28 \sim 0.82$ & \\
\hline $\mathrm{EL}(\mathrm{mm})^{\#}$ & $15.7 \pm 0.24^{\mathrm{a}}$ & $14.4 \pm 0.17^{\mathrm{a}}$ & $14.9 \pm 0.16^{\mathrm{a}}$ & $F_{2,187}=1.15, P=0.318$ \\
\hline range & $12.47 \sim 18.51$ & $11.49 \sim 19.50$ & $9.94 \sim 17.35$ & \\
\hline $\mathrm{EW}(\mathrm{mm})^{\#}$ & $8.4 \pm 0.08^{b}$ & $8.5 \pm 0.06^{\mathrm{a}}$ & $8.3 \pm 0.07^{\mathrm{b}}$ & $F_{2,187}=19.42, P<0.0001$ \\
\hline range & $7.19 \sim 9.03$ & $6.90 \sim 9.90$ & $6.39 \sim 9.36$ & \\
\hline $\mathrm{ES}^{\dagger}$ & $1.8 \pm 0.03^{\mathrm{a}}$ & $1.7 \pm 0.02^{\mathrm{b}}$ & $1.8 \pm 0.02^{\mathrm{ab}}$ & $F_{2,187}=6.71, r^{2}=0.06, P<0.0001$ \\
\hline Range & $1.44 \sim 2.27$ & $1.43 \sim 2.18$ & $1.47 \sim 2.11$ & \\
\hline $\mathrm{CS}^{\# *}$ & $2.9 \pm 0.13^{\mathrm{b}}$ & $3.7 \pm 0.18^{\mathrm{a}}$ & $3.8 \pm 0.14^{\mathrm{a}}$ & $F_{2,187}=10.93, P=0.0001$ \\
\hline range & $2 \sim 4$ & $2 \sim 6$ & $3 \sim 5$ & \\
\hline
\end{tabular}

